EUROPEAN JOURNAL OF PURE AND APPLIED MATHEMATICS

Vol. 15, No. 1, 2022, 207-223

ISSN 1307-5543 - ejpam.com

Published by New York Business Global

\title{
Restrained Disjunctive Domination in Graphs under some Binary Operations
}

\author{
Rolando P. Malalay ${ }^{1, *}$, Ferdinand P. Jamil ${ }^{2}$ \\ 1 College of Engineering and Technology, Zamboanga Peninsula Polytechnic State \\ University, 7000 Zamboanga City, Philippines \\ 2 Department of Mathematics and Statistics, College of Science and Mathematics, \\ Center of Graph Theory, Algebra and Analysis, Premier Research Institute of Science and \\ Mathematics, Mindanao State University-Iligan Institute of Technology, 9200 Iligan City, \\ Philippines
}

\begin{abstract}
A set $S \subseteq V(G)$ is a disjunctive dominating set of a graph $G$ if for every $v \in V(G) \backslash S, v$ is a neighbor of a vertex in $S$ or $S$ has at least two vertices each at distance 2 from $v$. We say that a disjunctive dominating set $S$ of $G$ is a restrained disjunctive dominating set if for each $v \in V(G) \backslash S$ there exists $u \in V(G) \backslash S$ such that $u v \in E(G)$ or there exist distinct vertices $u, w \in V(G) \backslash S$ such that $d_{G}(u, v)=2=d_{G}(w, v)$. The minimum cardinality $\gamma_{r}^{d}(G)$ of a restrained disjunctive dominating set of $G$ is the restrained disjunctive domination number of $G$. In this paper, we characterize the restrained disjunctive dominating sets in some binary operations such as the join, corona and lexicographic product of graphs and, as a result, obtain the values of their corresponding restrained disjunctive domination numbers.
\end{abstract}

2020 Mathematics Subject Classifications: 05C69

Key Words and Phrases: Disjunctive dominating set, restrained disjunctive dominating set, restrained disjunctive domination number, join, corona and lexicographic product

\section{Introduction}

The study of restrained domination in graphs was first initiated by Domke et al. [6] in 1997. Accordingly, they established the best possible upper and lower bounds for the restrained domination number of a connected graph $G$ and characterized those graphs achieving these bounds. In [16], the restrained dominating set of trees of order $n$ were characterized, and the exact values of the restrained domination number were determined. Some studies in restrained domination can be found in $[7,17,19]$.

On the other hand, disjunctive domination in graphs, specifically $b$-disjunctive, first introduced by Goddard et al. [8] in 2014 was motivated by the concept of domination

${ }^{*}$ Corresponding author.

DOI: https://doi.org/10.29020/nybg.ejpam.v15i1.4187

Email addresses: orlandomalalay@gmail.com (R. Malalay), ferdinand.jamil@g.msuiit.edu.ph (F. Jamil) 
in graphs (see $[4,10]$ ), the distance domination (see [5]) and the secondary domination (see [11]). While most of the variations on dominating sets which have been introduced recently tend to increase the domination number, which in effect raise implementation costs, disjunctive domination is a relaxation of the domination number [14]. In [8], sharp bounds for the disjunctive domination number were established for general graphs, and exact values were determined for specific graphs.

In 2016, Henning and Naicker also extended the concept of total domination by defining the disjunctive total domination. Accordingly, it allows for greater flexibility by modeling networks where one trades off redundancy and backup capability with resource optimization [14]. The above-mentioned authors established in [14] tight upper bound on the disjunctive total domination number of a graph in terms of its order and characterized the extremal graphs, and then proved that this bound can be significantly improved if claw-freeness of a graph is imposed. The same authors also investigated the variant on the class of trees in $[12,13]$.

Motivated by the concepts of $b$-disjunctive domination and disjunctive total domination in graphs, Jamil and Malalay [15] in 2019 extended the study of disjunctive dominating, particularly 2-disjunctive domination, and the disjunctive total dominating sets under some binary operations in graphs. Specifically, they characterized in [15] the disjunctive dominating sets and the disjunctive total dominating sets in the join, corona and lexicographic product of graphs and obtained the values of the respective corresponding disjunctive domination numbers.

In this paper, we introduce another variant of domination, namely restrained disjunctive domination, and characterize the restrained disjunctive dominating sets in the join, corona and lexicographic product of graphs.

In here, by a graph we mean a finite, simple and undirected connected graph $G=$ $(V(G), E(G))$. All basic terminologies used here are adapted from [2]. The symbols $V(G)$ and $E(G)$ are the vertex-set and edge-set, respectively, of $G$. For $S \subseteq V(G),|S|$ is the cardinality of $S$. In particular, $|V(G)|$ is called the order of $G$. The complement of $G$ is that graph $\bar{G}$, where $V(\bar{G})=V(G)$ and for its edges, $x y \in E(\bar{G})$ if and only if $x y \notin E(G)$.

Given graphs $G$ and $H$, the join of $G$ and $H$ is the graph $G+H$ with vertex set $V(G) \cup V(H)$ and edge set $E(G) \cup E(H) \cup\{u v: u \in V(G), v \in V(H)\}$. The corona of $G$ and $H$ is the graph $G \circ H$ obtained by taking one copy of $G$ and $|V(G)|$ copies of $H$, and then joining the $i^{t h}$ vertex of $G$ to every vertex in the $i^{\text {th }}$ copy of $H$. In particular, we call $G \circ K_{1}$ the corona of $G$, and write $\operatorname{cor}(G)=G \circ K_{1}$. The lexicographic product of $G$ and $H$ is the graph $G[H]$ with $V(G[H])=V(G) \times V(H)$ and $(u, v)\left(u^{\prime}, v^{\prime}\right) \in E(G[H])$ if and only if either $u u^{\prime} \in E(G)$ or $u=u^{\prime}$ and $v v^{\prime} \in E(H)$. In any of these graphs, $G$ and $H$ are referred to as their basic component graphs.

Vertices $u$ and $v$ of a graph $G$ are neighbors if $u v \in E(G)$. The open neighborhood of $v \in V(G)$ refers to the set $N_{G}(v)$ consisting of all neighbors of $v$. The degree of $v \in V(G)$ refers to the cardinality $\left|N_{G}(v)\right|$ of the open neighborhood of $v, \delta(G)$ is the minimum degree of a vertex of $G$, and $\Delta(G)$ is the maximum degree of $G$. The closed neighborhood of $v \in V(G)$ is $N_{G}[v]=N_{G}(v) \cup\{v\}$. Customarily, for $S \subseteq V(G), N_{G}(S)=\bigcup_{v \in S} N_{G}(v)$ 
and $N_{G}[S]=\bigcup_{v \in S} N_{G}[v]$. A subset $S \subseteq V(G)$ is a dominating set of $G$ if $N_{G}[S]=$ $V(G)$. In case, $S$ is a dominating set of $G$ and every vertex in $V(G) \backslash S$ is adjacent to another vertex in $V(G) \backslash S$, then $S$ is a restrained dominating set of $G$. The minimum cardinality $\gamma(G)$ of a dominating set of $G$ is the domination number of $G$, and the minimum cardinality $\gamma_{r}(G)$ of a restrained dominating set is the restrained domination number of $G$. A dominating set of cardinality $\gamma(G)$ is called a $\gamma$-set of $G$. Similarly, a $\gamma_{r}$-set is a restrained dominating set of cardinality $\gamma_{r}(G)$. The reader is referred to $[1,4,6,10,18]$ for the history, fundamental concepts and recent developments of domination in graphs as well as its various applications.

A dominating set $S \subseteq V(G)$ is a 2-dominating set of $G$ if for each $v \in V(G) \backslash S$, $\left|N_{G}(v) \cap S\right| \geq 2$. The minimum cardinality of a 2-dominating set is the 2-domination number of $G$, denoted by $\gamma_{\times 2}(G)$. Any 2-dominating set with cardinality $\gamma_{\times 2}(G)$ is called a $\gamma_{\times 2}$-set of $G$. The 2-domination in graphs is being studied in $[3,7,9]$.

For a vertex $v$ of $G, N_{G}(v, 2)=\left\{u \in V(G) \backslash\{v\}: d_{G}(u, v) \leq 2\right\}$. For $S \subseteq V(G)$, $N_{G}(S, 2)=\cup_{v \in S} N_{G}(v, 2)$. A set $S \subseteq V(G)$ is a distance-two dominating set of $G$ provided $V(G) \backslash S \subseteq N_{G}(S, 2)$, i.e., if for every $v \in V(G) \backslash S$ there exists $u \in S$ such that $d_{G}(u, v) \leq$ 2. The minimum cardinality $\gamma_{2}(G)$ of a distance-two dominating set is the distance-two domination number of $G$. A distance-two dominating set of cardinality $\gamma_{2}(G)$ is called a $\gamma_{2}$-set. Some studies in distance-two domination can be found in [20].

A set $S \subseteq V(G)$ is a disjunctive dominating set of $G$ if for every $v \in V(G) \backslash S, v$ is a neighbor of a vertex in $S$ or $S$ has at least two vertices each at distance 2 from $v$. The minimum cardinality of a disjunctive dominating set is the disjunctive domination number of $G$, and is denoted by $\gamma^{d}(G)$. A disjunctive dominating set of cardinality $\gamma^{d}(G)$ is called a $\gamma^{d}$-set. For convenience, the symbol $N_{G}^{d}(S)$ denotes the set of all $x \in V(G)$ such that $x y \in E(G)$ for some $y \in S$ or there exist distinct $u, v \in S$ with $d_{G}(x, u)=2=d_{G}(x, v)$. Then $S$ is a disjunctive dominating set of $G$ if and only if $V(G) \backslash S \subseteq N_{G}^{d}(S)$. Since $N_{G}(S) \subseteq N_{G}^{d}(S)$, dominating sets are disjunctive dominating sets. In particular, $\gamma^{d}(G)=$ 1 if and only if $\gamma(G)=1$.

A disjunctive dominating set $S$ of $G$ is a disjunctive total dominating set of $G$ provided $V(G)=N_{G}^{d}(S)$. That is, $S$ is a disjunctive total dominating set if for every $v \in S, v$ is adjacent to a vertex of $S$ or $S$ has at least two vertices each at distance 2 from $v$. The minimum cardinality of a disjunctive total dominating set of $G$ is the disjunctive total domination number of $G$, and is denoted by $\gamma_{t}^{d}(G)$. Any disjunctive total dominating set of cardinality $\gamma_{t}^{d}(G)$ is called $\gamma_{t}^{d}$-set.

Provided $G$ has no isolated vertices, a disjunctive dominating set $S$ of $G$ is a restrained disjunctive dominating set of $G$ if for each $v \in V(G) \backslash S$ there exists $u \in V(G) \backslash S$ such that $u v \in E(G)$ or there exist distinct vertices $u, w \in V(G) \backslash S$ such that $d_{G}(u, v)=$ $2=d_{G}(w, v)$. The minimum cardinality of a restrained disjunctive dominating set of $G$ is the restrained disjunctive domination number of $G$, and is denoted by $\gamma_{r}^{d}(G)$. Any restrained disjunctive dominating set of cardinality $\gamma_{r}^{d}(G)$ is called $\gamma_{r}^{d}$-set. Since restrained disjunctive dominating sets are disjunctive dominating sets, $1 \leq \gamma^{d}(G) \leq \gamma_{r}^{d}(G)$ for all graphs $G$ without isolated vertices. 


\section{Preliminaries and Known Results}

The following are some known results for paths and cycles.

Theorem 1. (i) [8] For all $n, \gamma^{d}\left(P_{n}\right)=\left\lceil\frac{n+1}{4}\right\rceil$;

(ii) [8] For cycle $C_{n}, n \geq 3, \gamma^{d}\left(C_{n}\right)=2$ for $n=4$, and $\gamma^{d}\left(C_{n}\right)=\left\lceil\frac{n}{4}\right\rceil$ for $n \neq 4$.

Next are our preliminary results which have exact values or bounds of some special graphs in terms of the restrained disjunctive domination number. Let $G$ be a connected graph and $u, v \in V(G)$ such that $u v \in E(G)$. If $u$ is the end vertex of graph $G$ and $\operatorname{deg}_{G}(v) \leq 2$, then $u \in S$ for all restrained disjunctive dominating sets $S$ of $G$.

Proposition 1. (i) For path $P_{n}, n \geq 1$,

$$
\gamma_{r}^{d}\left(P_{n}\right)= \begin{cases}3, & \text { if } n=3 \\ \left\lceil\frac{n-1}{4}\right\rceil+1, & \text { if } n \neq 3 .\end{cases}
$$

(ii) For cycle $C_{n}, n \geq 3$,

$$
\gamma_{r}^{d}\left(C_{n}\right)= \begin{cases}1, & \text { if } n=3 \\ 2, & \text { if } n=4 \\ \left\lceil\frac{n}{4}\right\rceil, & \text { if } n \geq 5\end{cases}
$$

Proof. To prove $(i)$, let $P_{n}=\left[x_{1}, x_{2}, \ldots, x_{n}\right]$. The case where $1 \leq n \leq 4$ is obvious. Suppose that $n \geq 5$. Let $k$ be the largest integer for which $4 k+1 \leq n$. If $n-4 k-1 \neq 2$, take $S=\left\{x_{1}, x_{5}, \ldots, x_{4 k+1}, x_{n}\right\}$. Otherwise, take $S=\left\{x_{1}, x_{5}, \ldots, x_{4 k}, x_{n}\right\}$. Then $S$ is a restrained disjunctive dominating set of $P_{n}$. Thus, $\gamma_{r}^{d}\left(P_{n}\right) \leq\left\lceil\frac{n-1}{4}\right\rceil+1$. Now, suppose that $S^{*} \subseteq V(G)$ is a restrained disjunctive dominating set of $G$ with $\left|S^{*}\right| \leq\left\lceil\frac{n-1}{4}\right\rceil$. In view of Observation $2, x_{1}, x_{n} \in S^{*}$. In particular, $\left|S^{*}\right|=1$ if $n=5$, which is impossible. Let $n \geq 6$. Pick $u, v \in S$ such that $d_{P_{n}}(u, v)$ is maximum among all pairs $(x, y) \in S \times S$ for which $S \backslash\{x, y\}$ does not contain a vertex lying in the $x-y$ path. Then $d_{P_{n}}(u, v) \geq 5$. Thus, there exists $w \in V\left(P_{n}\right) \backslash S$ such that $w \notin N_{P_{n}}^{d}(S)$, a contradiction.

The case where $3 \leq n \leq 4$ is obvious for $C_{n}$. Let $n \geq 5$. By Theorem $1,\left\lceil\frac{n}{4}\right\rceil=$ $\gamma^{d}\left(C_{n}\right) \leq \gamma_{r}^{d}\left(C_{n}\right)$. Let $C_{n}=\left[x_{1}, x_{2}, \ldots, x_{n}, x_{1}\right]$. Define $S=\left\{x_{1}, x_{5}, \ldots, x_{4 k+1}\right\}$, where $k$ is the largest integer for which $4 k+1 \leq n$. Then $S$ is a restrained disjunctive dominating set of $C_{n}$. Thus, $\gamma_{r}^{d}\left(C_{n}\right) \leq|S|=\left\lceil\frac{n}{4}\right\rceil$.

Theorem 2. Let $G$ be a connected graph of order $n \geq 2$. Then

(i) $\gamma_{r}^{d}(G) \leq n-\Delta(G)$ provided $\Delta(G) \geq 3$.

(ii) $\gamma_{r}^{d}(G)=1$ if and only if $n \geq 3, G \neq P_{3}$ and $\gamma(G)=1$. 
Proof. To prove $(i)$, suppose that $\Delta(G) \geq 3$, and let $v \in V(G)$ for which $\operatorname{deg}_{G}(v)=$ $\Delta(G)$. Put $S=V(G) \backslash N_{G}(v)$. Then $S$ is a restrained disjunctive dominating set of $G$, and the result follows.

To prove (ii), suppose that $\gamma_{r}^{d}(G)=1$. In view of the remark above, $1 \leq \gamma^{d}(G) \leq$ $\gamma_{r}^{d}(G)=1$ showing that $\gamma^{d}(G)=1$. By the remark above, $\gamma(G)=1$. Since $\gamma_{r}^{d}\left(P_{2}\right)=2$, $n \geq 3$. In the case where $n=3, G=K_{3}$. To prove the converse, it is enough to consider only the case where $n \geq 4$. Let $S=\{v\}$ be a dominating set of $G$. Then $S$ is a disjunctive dominating set of $G$. Let $u \in V(G) \backslash S$. Since $S$ is a dominating set, $u v \in E(G)$. Since $n \geq 4$, we can pick distinct $x, y \in V(G) \backslash\{u, v\}$. Then $v x \in E(G)$ and $v y \in E(G)$. If $u x \in E(G)$ or $u v \in E(G)$, then we are done. Suppose that $u x, u y \notin E(G)$. Then $[u, v, x]$ is a $u-x$ geodesic in $G$. Thus, $d_{G}(u, x)=2$. Similarly, $d_{G}(u, y)=2$. This shows that $S$ is a restrained disjunctive dominating set of $G$. Accordingly, $\gamma_{r}^{d}(G)=1$.

Corollary 1. For a connected graph $G$ of order $n \geq 3, \gamma_{r}^{d}(G)=1$ if and only if $G=K_{3}$ or $G=K_{1}+H$ for some graph $H$ with $|V(H)| \geq 3$.

Note that since $\gamma_{r}^{d}\left(K_{1, n}\right)=1$ for all $n \geq 3$, the bound in Theorem $2(i)$ is sharp.

\section{Exploratory Examples}

For any connected graph $G, \gamma_{r}^{d}(G) \leq \gamma_{r}(G)$ and $\gamma^{d}(G) \leq \gamma_{r}^{d}(G)$, and the following example shows that up to some extent, the values of these parameters can be pre-assigned.

Example 1. For any positive integers $a, b$ with $2 \leq a \leq b$ where $b \leq 3 a-1$, there exists a connected graph $G$ such that $\gamma_{r}^{d}(G)=a$ and $\gamma_{r}(G)=b$.

To see this, consider the following cases:

Case 1. $a=b$

In this case, we take $G$ as the graph $G_{1}$ obtained by connecting a copies of 3-pan graph using the path $\left[u_{1}, u_{2}, \ldots, u_{a}\right]$, where $u_{i}$ is a pendant of the $i^{\text {th }}$ copy of 3-pan graph for each $i \in\{1,2, \ldots, a\}$ as shown in Figure 1. Denote by $P^{u}$ that copy of 3-pan graph corresponding to vertex $u$.

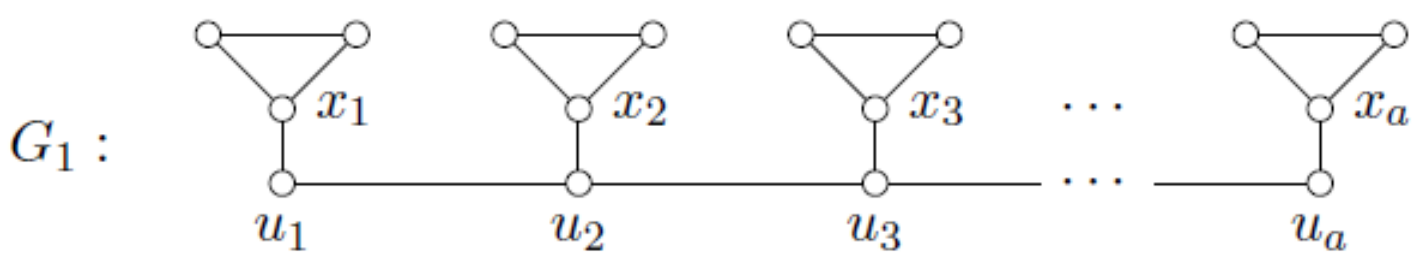

Figure 1: A graph $G$ with $2 \leq \gamma_{r}^{d}(G)=\gamma_{r}(G)$

Since the set $S=\left\{x_{i}: i \in\{1,2, \ldots, a\}\right\}$ is both a restrained disjunctive dominating set and a restrained dominating set of $G, \gamma_{r}^{d}(G)=\gamma_{r}(G) \leq|S|=a$. Let $D \subseteq V(G)$ 
such that $|D|<a$. Then there exists $1 \leq k \leq a$ such that $D \cap V\left(3-P^{u_{k}}\right)=\varnothing$. In particular, $V\left(P^{u_{k}}\right) \backslash\left\{x_{k}, u_{k}\right\}$ is not dominated by $D$ in the sense of disjunctive domination and the usual domination. Thus, $\gamma_{r}^{d}(G) \geq a$ and $\gamma_{r}(G) \geq a$. Consequently, $\gamma_{r}^{d}(G)=a$ and $\gamma_{r}(G)=a=b$.

Case 2: $a<b$

Here, we write $b=a+k$, where $k \geq 1$. Obtain the graph $G^{*}$ as graph $G_{2}$ from graph $G_{1}$ in the first case by adding to $G_{1}$ the pendant edges $x_{1} y_{i}$ for each $i \in\{1,2, \ldots, k\}$ as shown in Figure 2.

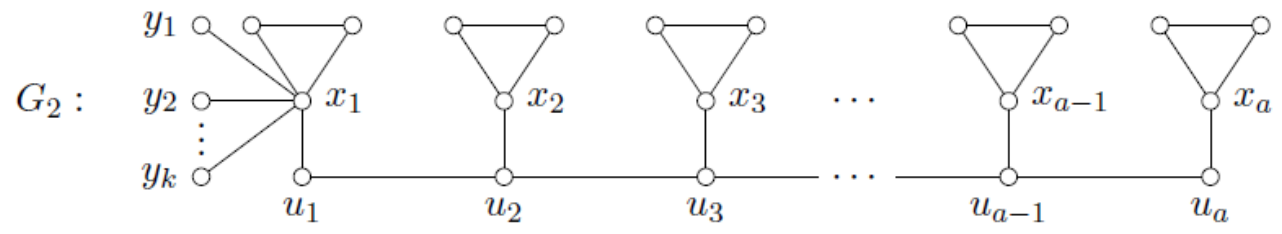

Figure 2: A graph $G^{*}$ with $2 \leq \gamma_{r}^{d}\left(G^{*}\right)<\gamma_{r}\left(G^{*}\right)$

Since $S_{1}=\left\{x_{i}: i \in\{1,2, \ldots, a\}\right\}$ is a $\gamma_{r}^{d}$-set of $G^{*}, \gamma_{r}^{d}\left(G^{*}\right)=\left|S_{1}\right|=a$. Observe that the set $S_{2}=S_{1} \cup\left\{y_{j}: j \in\{1,2, \ldots, k\}\right\}$ is a restrained dominating set of $G^{*}$ showing that $\gamma_{r}\left(G^{*}\right) \leq\left|S_{2}\right|=a+k=b$. Let $S \subseteq V\left(G^{*}\right)$ be a $\gamma_{r}$-set of $G^{*}$. Then $S$ contains $S_{1}$. Being a restrained disjunctive dominating set of $G^{*}$ and $S$ contains $S_{1}$, necessarily $y_{j} \in S$, for each $j \in\{1,2, \ldots, k\}$. Thus, $\gamma_{r}\left(G^{*}\right) \geq|S|=a+k=b$. Consequently, $\gamma_{r}\left(G^{*}\right)=b$.

Example 2. For a pair of positive integers $(a, b)$ with $2 \leq a \leq b$ where either $b \leq \frac{3 a}{2}$ if $a$ is even or $b \leq \frac{3 a+1}{2}$ when $a$ is odd, there exists a connected graph $G$ such that $\gamma^{\bar{d}}(G)=a$ and $\gamma_{r}^{d}(G)=b$.

Consider the following cases:

Case 1. $a=b$

Take $G$ to be the graph $G_{1}$ obtained from the path $P_{2 a-1}=\left[u_{1}, u_{2}, \ldots, u_{2 a-1}\right]$ as shown in Figure 3, by adding to $P_{2 a-1}$ the pendant edges $x_{j} u_{2 j-1}$ for all $j=1,2, \ldots, a$. The

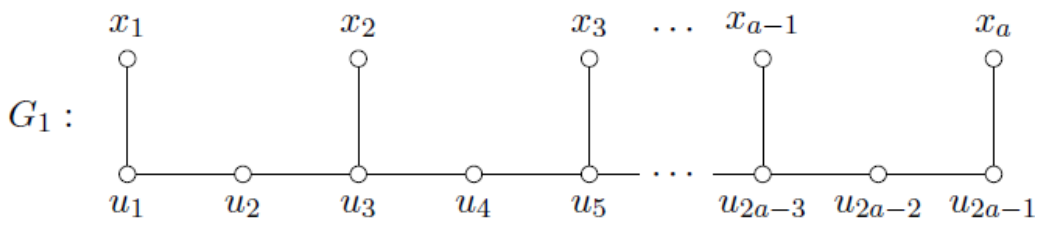

Figure 3: A graph $G$ with $\gamma^{d}(G)=\gamma_{r}^{d}(G)$

set $\left\{x_{i}: i=1,2, \ldots, a\right\}$ is both a disjunctive dominating set and a restrained disjunctive dominating of $G$. Thus, $\gamma^{d}(G) \leq a$ and $\gamma_{r}^{d}(G) \leq a=b$. Let $S \subseteq V(G)$ be a $\gamma^{d}$-set of $G$. Then either $x_{j} \in S$ or $u_{2 j-1} \in S$ for all $j=1,2, \ldots, a$. Also, being a disjunctive dominating set of $G$, if $u_{2 j-2} \in S$ for all $j=2,3, \ldots, a$, then necessarily $u_{1} u_{2 a-1} \in S$. 
This means that $\gamma^{d}(G) \geq a$. Thus, $\gamma^{d}(G)=a$. Next, if $S \subseteq V(G)$ is a $\gamma_{r}^{d}$-set of $G$, then $x_{i} \in S$ for all $i=1,2, \ldots, a$ or $x_{1} x_{a} \in S$ and $u_{2 j-1} \in S$ for all $j=2,3, \ldots, a-1$. Being a restrained disjunctive dominating set of $G$, if $u_{2 j-2} \in S$ for all $j=2,3, \ldots, a$, then necessarily $u_{1}, x_{1}, u_{2 a-1}, x_{a} \in S$. Similarly, if $u_{2 j-1} \in S$ for all $j=1,2, \ldots, a$, then necessarily $x_{1}, x_{a} \in S$. Moreover, if $x_{j} u_{2 j-1} \in S$ for all $j=1,2, \ldots, a$, then $u_{2 i-2} \in S$ for all $i=2,3, \ldots, a$. In any case, $\gamma_{r}^{d}(G) \geq a$. Thus, $\gamma_{r}^{d}(G)=a=b$.

Case 2. $a<b$

If $a$ is even, then by hypothesis, $b \leq \frac{3 a}{2}=a+\frac{a}{2}$. Write $b=a+k$, where $1 \leq k \leq \frac{a}{2}$. Take $G=G_{2}$ obtained from $G_{1}$ by adding pendant edges $x_{i} y_{i}$ for all $i=1,2, \ldots, 2 k-1$ as shown in Figure 4. Since the set $\left\{x_{i}: i=1,2, \ldots, a\right\}$ is a $\gamma^{d}$-set of $G, \gamma^{d}(G)=a$.

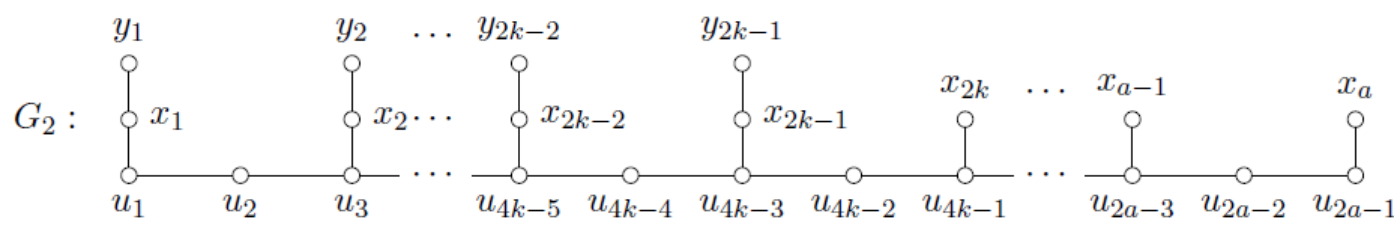

Figure 4: A graph $G$ with $\gamma^{d}(G)<\gamma_{r}^{d}(G)$

Next, to determine the restrained disjunctive domination number of $G$, first consider the case where $4 k-2=2 a-2$. Let $S=\left\{x_{i}, y_{j}: i=2 k, 2 k+1, \ldots, a ; j=1,2, \ldots, 2 k-\right.$ $1\} \cup\left\{u_{4 k-4}, u_{2(2 j-1)}: j=1,2, \ldots, k-1\right\}$. Then $S$ is a restrained disjunctive dominating set of $G$. Hence, $\gamma_{r}^{d}(G) \leq|S|=[a-(2 k-1)]+(2 k-1)+[(k-1)+1]=a+k=b$. Let $S \subseteq V(G)$ be a $\gamma_{r}^{d}$-set of $G$. Necessarily, $x_{a}, y_{j} \in S$ for each $j \in\{1,2, \ldots, 2 k-1\}$. Observe that, if $x_{i} \in S$ for each $i \in\{1,2, \ldots, a-1\}$ or $u_{2 a-1}, u_{2(2 j-1)} \in S$ for each $j \in\{1,2, \ldots, k\}$, then $|S| \geq a+k+1$ which is a contradiction. This implies that the set $\left\{x_{i}, y_{j}: i=2 k, 2 k+1, \ldots, a ; j=1,2, \ldots, 2 k-1\right\} \cup\left\{u_{4 k-4}, u_{2(2 j-1)}: j=1,2, \ldots, k-1\right\}$ is a $\gamma_{r}^{d}$-set of $G$. Thus, $\gamma_{r}^{d}(G)=a+k=b$. Suppose that $4 k-2<2 a-2$. Let $S=\left\{x_{i}, y_{j}: i=\right.$ $2 k, 2 k+1, \ldots, a ; j=1,2, \ldots, 2 k-1\} \cup\left\{u_{2(2 j-1)}: j=1,2, \ldots, k\right\}$. Then $S$ is a restrained disjunctive dominating set of $G$. Thus, $\gamma_{r}^{d}(G) \leq|S|=[a-(2 k-1)]+(2 k-1)+k=a+k=b$. Following similar arguments as above show that $\gamma_{r}^{d}(G)=b$.

Now, suppose that $a$ is odd. Then $b \leq \frac{3 a+1}{2}=a+\frac{a+1}{2}$. Let $b=a+k$, where $1 \leq k \leq \frac{a+1}{2}$. If $4 k-2=2 a$, then $\gamma_{r}^{d}(G)=b$ as determined by the set $\left\{x_{i}, y_{j}: i=2 k, 2 k+\right.$ $1, \ldots, a ; j=1,2, \ldots, 2 k-1\} \cup\left\{u_{4 k-4}, u_{2(2 j-1)}: j=1,2, \ldots, k-1\right\}$ and if $4 k-2<2 a$, then $S=\left\{x_{i}, y_{j}: i=2 k, 2 k+1, \ldots, a ; j=1,2, \ldots, 2 k-1\right\} \cup\left\{u_{2(2 j-1)}: j=1,2, \ldots, k\right\}$ is a $\gamma_{r}^{d}$-set of $G$. Thus, $\gamma_{r}^{d}(G)=b$.

\section{Main Results}

\subsection{On join of graphs}

Theorem 3. [15] Let $G$ and $H$ be any graphs, and $S \subseteq V(G+H)$. Then $S$ is a disjunctive dominating set of $G+H$ if and only if one of the following holds: 
(i) $S \subseteq V(G)$ for which either $|S| \geq 2$ or $S=\{x\}$ where $N_{G}[x]=V(G)$.

(ii) $S \subseteq V(H)$ for which either $|S| \geq 2$ or $S=\{x\}$ where $N_{H}[x]=V(H)$.

(iii) $S \cap V(G) \neq \varnothing$ and $S \cap V(H) \neq \varnothing$.

For any nontrivial graphs $G$ and $H$, if $S \subseteq V(G+H)$ intersects both $V(G)$ and $V(H)$, then $S$ is a (restrained) dominating set, hence is a (restrained) disjunctive dominating set of $G+H$.

For any graph $G$,

$$
\gamma_{r}^{d}\left(G+K_{1}\right)= \begin{cases}1, & \text { if } G=K_{2} \text { or }|V(G)| \geq 3 \\ 2, & \text { if } G=K_{1} \\ 3, & \text { if } G=\overline{K_{2}} .\end{cases}
$$

Theorem 4. Let $G$ and $H$ be nontrivial connected graphs. Then $S \subseteq V(G+H)$ is a restrained disjunctive dominating set of $G+H$ if and only if one of the following holds;

(i) $S \subseteq V(G)$ and either $|S| \geq 2$ or $S=\{x\}$ where $N_{G}[x]=V(G)$;

(ii) $S \subseteq V(H)$ and either $|S| \geq 2$ or $S=\{x\}$ where $N_{H}[x]=V(H)$; and

(iii) S intersects both $V(G)$ and $V(H)$ satisfying the following:

(a) If $V(G) \subseteq S$, then $V(H) \subseteq S$ or $\langle V(H) \backslash S\rangle \cong K_{2}$ or $|V(H) \backslash S| \geq 3$.

(b) If $V(H) \subseteq S$, then $V(H) \subseteq S$ or $\langle V(H) \backslash S\rangle \cong K_{2}$ or $|V(H) \backslash S| \geq 3$.

Proof. Let $S \subseteq V(G+H)$ be a restrained disjunctive dominating set of $G+H$. Then $S$ is a disjunctive dominating set of $G+H$. By Theorem 3, if $S \subseteq V(G)$, then either $|S| \geq 2$ or $S=\{x\}$ where $N_{G}[x]=V(G)$. Similarly, if $S \subseteq V(H)$, then either $|S| \geq 2$ or $S=\{x\}$ where $N_{H}[x]=V(H)$. Now suppose that $S$ intersects both $V(G)$ and $V(H)$. Suppose further that $V(G) \subseteq S$. Let $v \in V(H) \backslash S$. Since $S$ is a restrained disjunctive dominating set, there exists $u \in V(H) \backslash S$ such that $u v \in E(G+H)$ or there exist distinct $u, w \in V(H) \backslash S$ such that $d_{G+H}(u, v)=2=d_{G+H}(w, v)$. If the former holds, then $V(H) \subseteq S$ or $\langle V(H) \backslash S\rangle \cong K_{2}$. If the latter holds, then $|V(H) \backslash S| \geq 3$. Similarly, if $V(H) \subseteq S$, then $(b)$ holds.

Conversely, suppose that $(i)$ holds for $S$. By Theorem $3, S$ is a disjunctive dominating set of $G+H$. Let $v \in V(G+H) \backslash S$. If $v \in V(G)$, then $u v \in E(G+H)$ for any $u \in V(H)$. If $v \in V(H)$, then pick any $u \in V(H)$ for which $u v \in E(H)$. Accordingly, $S$ is a restrained disjunctive dominating set of $G+H$. Similarly, if $(i i)$ holds for $S$, then $S$ is a restrained disjunctive dominating set of $G+H$. Suppose that (iii) holds for $S$. By Theorem $3, S$ is a disjunctive dominating set of $G+H$. If $V(G) \backslash S \neq \varnothing$ and $V(H) \backslash S \neq \varnothing$, then the conclusion follows because $x y \in E(G+H)$ for all $x \in V(G)$ and for all $y \in V(H)$. Suppose that $V(G) \subseteq S$, and let $v \in V(G+H) \backslash S$. Since $V(H) \subseteq S$ or $\langle V(H) \backslash S\rangle \cong K_{2}$, $v \in V(H) \backslash S$. Suppose that, there does not exist $u \in V(H) \backslash S$ for which $u v \in E(H)$, 
then $|V(H) \backslash S| \geq 3$. In particular, $|V(H) \backslash(S \cup\{v\})| \geq 2$, say $u, w \in V(H) \backslash(S \cup\{v\})$, with $u \neq w$. Then $d_{G+K}(u, v)=2=d_{G+H}(w, v)$. Similarly, if $V(H) \subseteq S$, then for each $v \in V(G+H) \backslash S$, there exists $u \in V(G) \backslash S$ for which $u v \in E(G+H)$ or there exist distinct $u, w \in V(G) \backslash S$ such that $d_{G+H}(u, v)=2=d_{G+H}(w, v)$. Accordingly, $S$ is a restrained disjunctive dominating set of $G+H$.

Corollary 2. For nontrivial connected graphs $G$ and $H$,

$$
\gamma_{r}^{d}(G+H)= \begin{cases}1, & \text { if } \gamma(G)=1 \text { or } \gamma(H)=1 \\ 2, & \text { otherwise }\end{cases}
$$

Proof. Suppose that $\gamma(G)=1$, and let $S=\{v\}$ be a dominating set of $G$. Then $S$ is a restrained disjunctive dominating set of $G+H$ by Theorem 4 . In this case, $\gamma_{r}^{d}(G+H)=1$. Similarly, if $\gamma(H)=1$, then $\gamma_{r}^{d}(G+H)=1$. Suppose that $\gamma(G) \geq 2$ and $\gamma(H) \geq 2$. In view of Theorem $4, \gamma_{r}^{d}(G+H) \geq 2$. Pick $u \in V(G)$ and $v \in V(H)$. By Theorem $4,\{u, v\}$ is a restrained disjunctive dominating set of $G+H$. Thus, $\gamma_{r}^{d}(G+H) \leq 2$.

\subsection{On corona of graphs}

It is worth noting that $G \circ H$ is composed of the subgraphs $H^{v}+\langle v\rangle$ joined together by the edges of $G$. Thus,

$$
V(G \circ H)=V(G) \cup\left(\cup_{v \in V(G)} V\left(H^{v}\right)\right)=\cup_{v \in V(G)} V\left(H^{v}+v\right) .
$$

Theorem 5. [15] Let $G$ be a nontrivial connected graph and $H$ be any graph, and let $S \subseteq V(G \circ H)$. Then $S$ is a disjunctive dominating set of $G \circ H$ if and only if each of the following holds for $S$ :

(i) $\left|S \cap N_{G}(v)\right| \geq 2$ for all $v \in V(G) \backslash S$ with $S \cap V\left(H^{v}\right)=\varnothing$;

(ii) $\left|S \cap V\left(H^{v}\right)\right| \geq 1$ for all $v \in V(G) \backslash S$ with $\left|S \cap N_{G}(v)\right|=1$; and

(iii) $S \cap V\left(H^{v}\right)$ is a disjunctive dominating set of $H^{v}+v$ for all $v \in V(G) \backslash S$ with $S \cap N_{G}(v)=\varnothing$. In particular, if $\gamma(H)>1$, then $\left|S \cap V\left(H^{v}\right)\right| \geq 2$.

Corollary 3. [15] Let $G$ be a nontrivial connected graph. Then for any graph $H, \gamma^{d}(G \circ$ $H)=\gamma_{\times 2}(G)$.

Proposition 2. Let $G$ be a nontrivial connected graph and $S \subseteq V\left(G \circ K_{1}\right)$. Then $S$ is a restrained disjunctive dominating set of $G \circ K_{1}$ if and only if each of the following holds:

(i) $u^{v} \in S$ for all $v \in \operatorname{End}(G)$;

(ii) For each $u^{v} \notin S$,

(a) $\left|N_{G}(v) \cap S\right| \geq 2$ whenever $v \notin S ;$ 
(b) $\left|N_{G}(v) \backslash S\right| \geq 2$ whenever $v \in S$.

(iii) For each $v \notin S$ with $N_{G}(v) \cup\left\{u^{v}\right\} \subseteq S$,

(a) $\left|N_{G}(v, 2) \backslash S\right| \geq 2$ whenever $u^{w} \in S$ for all $w \in N_{G}(v)$; and

(b) $\left|N_{G}(v, 2) \backslash S\right| \geq 1$ whenever $u^{w} \notin S$ for exactly one $w \in N_{G}(v)$. In particular, $v \in \operatorname{End}(G)$ if and only if $\left|N_{G}(v)\right|=1$.

Proof. Let $S \subseteq V\left(G \circ K_{1}\right)$ be a restrained disjunctive dominating set of $G \circ K_{1}$. Let $v \in$ $\operatorname{End}(G)$. Suppose that $u^{v} \notin S$. If $v \in S$, then since $S$ is a restrained disjunctive dominating set, there exist distinct $w, z \in N_{G}(v) \backslash S$ for which $d_{G \circ K_{1}}\left(u^{v}, w\right)=2=d_{G \circ K_{1}}\left(u^{v}, z\right)$, and consequently, $d_{G}(w, v)=1=d_{G}(z, v)$. This is impossible since $v \in \operatorname{End}(G)$. If $v \notin S$, then since $S$ is a disjunctive dominating set, there exist distinct $w, z \in N_{G}(v) \cap S$ for which $d_{G \circ K_{1}}\left(u^{v}, w\right)=2=d_{G \circ K_{1}}\left(u^{v}, z\right)$, which for the same reason is impossible. Thus, $u^{v} \in S$ and $(i)$ holds. Let $u^{v} \notin S$. By $(i), v \notin \operatorname{End}(G)$. If $v \notin S$, then since $S$ is a disjunctive dominating set, there exist distinct $w, z \in N_{G}(v) \cap S$ for which $d_{G \circ K_{1}}\left(u^{v}, w\right)=$ $2=d_{G \circ K_{1}}\left(u^{v}, z\right)$. Thus, $\left|N_{G}(v) \cap S\right| \geq 2$ and Property $(i i)(a)$ holds for $S$. On the other hand, if $v \in S$, then since $S$ is a restrained disjunctive dominating set, there exist distinct $w, z \in V(G) \backslash S$ for which $d_{G \circ K_{1}}\left(w, u^{v}\right)=2=d_{G \circ K_{1}}\left(z, u^{v}\right)$. This proves that Property (ii)(b) holds for $S$. Now, let $v \in V(G) \backslash S$ with $N_{G}(v) \cup\left\{u^{v}\right\} \subseteq S$. Suppose that $u^{w} \in S$ for all $w \in N_{G}(v)$. Since $S$ is a restrained disjunctive dominating set, there exist distinct $w, z \in S$ for which $d_{G \circ K_{1}}(v, w)=2=d_{G \circ K_{1}}(v, z)$. The hypothesis implies that $w, z \in V(G)$, proving $(i i i)(a)$. Similarly, $(i i i)(b)$ holds for $S$.

Conversely, suppose that all the properties hold for $S$. First, using Theorem 5 we prove that $S$ is a disjunctive dominating set of $G \circ K_{1}$. Let $v \in V(G) \backslash S$. By Property (ii) (a), if $u^{v} \notin S$, then $\left|S \cap N_{G}(v)\right| \geq 2$. By the same property, if $\left|S \cap N_{G}(v)\right|=1$, then $u^{v} \in S$ so that $\left|S \cap V\left(K_{1}^{v}\right)\right|=1$. Now suppose that $S \cap N_{G}(v)=\varnothing$. Note that either $u^{v} \in S$ or $u^{v} \notin S$. If $u^{v} \notin S$, then by Property $(i i)(a),\left|S \cap N_{G}(v)\right| \geq 2$, a contradiction. Thus, $u^{v} \in S$ and $S \cap V\left(K_{1}^{v}\right)=\left\{u^{v}\right\}$ is a disjunctive dominating set of $K_{1}^{v}+v$. Therefore, $S$ is a disjunctive dominating set of $G \circ K_{1}$. Now, we show that $S$ is a restrained disjunctive dominating set. Let $x \in V\left(G \circ K_{1}\right) \backslash S$. Suppose that $x=u^{v}$ for some $v \in V(G)$. If $v \notin S$, then we are done. If $v \in S$, then we pick distinct $w, z \in N_{G}(v) \backslash S$, as guaranteed by Property $(i i)(b)$. Then $d_{G \circ K_{1}}(x, w)=2=d_{G \circ K_{1}}(x, z)$. Suppose that $x \in V(G)$. If $u^{x} \notin S$ or $N_{G}(x) \backslash S \neq \varnothing$, then we are done. Suppose that $u^{x} \in S$ and $N_{G}(x) \subseteq S$. Properties $(i i i)(a)$ and $(i i i)(b)$ imply that there exists distinct $w, z \in V\left(G \circ K_{1}\right) \backslash S$ for which $d_{G \circ K_{1}}(x, w)=2=d_{G \circ K_{1}}(x, z)$.

Corollary 4. For a nontrivial connected graph $G$,

$$
|\operatorname{End}(G)| \leq \gamma_{r}^{d}\left(G \circ K_{1}\right) \leq|V(G)|,
$$

and these bounds are sharp.

Proof. The left-hand inequality follows immediately from Property $(i)$ of Proposition 2 for any restrained disjunctive dominating set. Let $S=\left\{u^{v}: v \in V(G)\right\}$. Then $S$ is 
a restrained disjunctive dominating set of $G \circ K_{1}$ by Proposition 2, and the right-hand inequality follows. For the sharpness of the bounds, verify that $\gamma_{r}^{d}\left(K_{1, n} \circ K_{1}\right)=n=$ $\left|\operatorname{End}\left(K_{1, n}\right)\right|$ for any $n \geq 2$ and $\gamma_{r}^{d}\left(P_{3} \circ K_{1}\right)=3=\left|V\left(P_{3}\right)\right|$.

Corollary 5. (i) For $n \geq 3, \gamma_{r}^{d}\left(P_{n} \circ K_{1}\right)=\left\lfloor\frac{n}{2}\right\rfloor+2$,

(ii) For $n \geq 3$,

$$
\gamma_{r}^{d}\left(C_{n} \circ K_{1}\right)= \begin{cases}\left\lfloor\frac{n}{2}\right\rfloor, & \text { if } n \equiv 0(\bmod 2) \\ \left\lfloor\frac{n}{2}\right\rfloor+2, & \text { otherwise; }\end{cases}
$$

and

(iii) For $m, n \geq 2, \gamma_{r}^{d}\left(K_{m, n} \circ K_{1}\right)=\min \{m, n, 4\}$.

Proof. Let $P_{n}=\left[x_{1}, x_{2}, \ldots, x_{n}\right]$ and $k=\left\lfloor\frac{n}{2}\right\rfloor$. Since by Proposition 2 , the set $\left\{u^{x_{1}}, x_{2}, x_{4}, \ldots, x_{2 k}, u^{x_{n}}\right\}$ is a restrained dominating set, $\gamma_{r}^{d}\left(P_{n}\right) \leq k+2$. Suppose that $S \subseteq V\left(G \circ K_{1}\right)$ is a restrained disjunctive dominating set of $G \circ K_{1}$ with $|S| \leq k+1$. Accordingly, $\left\{u^{x_{1}}, u^{x_{n}}\right\} \subseteq S$. Apparently, there exist $w, v \in V\left(P_{n}\right)$ for which $w v \in E(G)$ and all vertices $w, v, u^{v}, u^{w} \notin S$. In particular, $\left|N_{G}(v) \cap S\right| \geq 2$ by Proposition 2 , which is impossible since $w \notin S$. Thus, $\gamma_{r}^{d}\left(P_{n} \circ K_{1}\right) \geq k+2$. This proves Property $(i)$. To prove $(i i)$, let $C_{n}=\left[x_{1}, x_{2}, \ldots, x_{n}\right]$. Let $k=\left\lfloor\frac{n}{2}\right\rfloor$. If $n=2 k$, then the set $\left\{x_{2}, x_{4}, \ldots, x_{2 k}\right\}$ is a restrained disjunctive dominating set of $C_{n} \circ K_{1}$ so that $\gamma_{r}^{d}\left(C_{n} \circ K_{1}\right) \leq k$. On the other hand, if $n=2 k+1$, then the set $\left\{u^{x_{1}}, x_{2}, x_{4}, \ldots, x_{2 k}, u^{x_{n}}\right\}$ is a restrained disjunctive dominating set of $C_{n} \circ K_{1}$, and so $\gamma_{r}^{d}\left(C_{n} \circ K_{1}\right) \leq k+2$. Following similar arguments as in the proof for $P_{n}$, the desired equality is attained. Let $U$ and $V$ be the partite sets of $K_{m, n}$ with $|U|=m$ and $|V|=n$. By Proposition $2, U$ or $V$ are restrained disjunctive dominating sets of $K_{m, n} \circ K_{1}$. Suppose that $m \geq 4$ and $n \geq 4$. Pick $x, y \in U$ and $a, b \in V$. By Proposition 2, $S=\{x, y, a, b\}$ is a restrained disjunctive dominating set of $K_{m, n} \circ K_{1}$. Thus,

$$
\gamma_{r}^{d}\left(K_{m, n} \circ K_{1}\right) \leq \min \{m, n, 4\} .
$$

Now, let $S \subseteq V\left(K_{m, n} \circ K_{1}\right)$ be a restrained disjunctive dominating set of $K_{m, n} \circ K_{1}$. Suppose that $|S| \leq 3$. Then there exists $v \in V\left(K_{m, n} \circ K_{1}\right)$ for which $u^{v} \in S$. In view of Proposition 2, this is only possible if $S=U$ or $S=V$. Thus,

$$
\gamma_{r}^{d}\left(K_{m, n} \circ K_{1}\right) \geq \min \{m, n, 4\} .
$$

Theorem 6. Let $G$ and $H$ be nontrivial connected graphs, and let $S \subseteq V(G \circ H)$. Then $S$ is a restrained disjunctive dominating set of $G \circ H$ if and only if $S$ satisfies all the properties in Theorem 5 as well as each of the following properties:

(i) For each $v \in S \cap V(G)$, at least one of the following holds: 
(a) $S \cap V\left(H^{v}+v\right)$ is a restrained disjunctive dominating set of $H^{v}+v$ whenever $N_{G}(v) \subseteq S$; or

(b) $\left|V\left(H^{v}\right) \backslash S\right| \geq 2$ whenever $\left|N_{G}(v) \backslash S\right|=1$ and $V\left(H^{v}\right) \backslash S \neq \varnothing$.

(ii) For each $v \in V(G) \backslash S$ for which $S \cap V\left(H^{v}\right) \neq \varnothing$, at least one of the following holds:

(a) $V\left(H^{v}\right) \backslash S \neq \varnothing$;

(b) $N_{G}(v) \backslash S \neq \varnothing$; or

(c) $\left|N_{G}(v, 2) \cap[V(G) \backslash S]\right| \geq 2$.

Proof. Suppose that $S$ is a restrained disjunctive dominating set of $G \circ H$. Since $S$ is a disjunctive dominating set, all properties in Theorem 5 hold for $S$. To prove $(i)$, let $v \in S \cap V(G)$. Suppose that $N_{G}(v) \subseteq S$. Since $V\left(H^{v}\right) \subseteq N_{H^{v}+v}(v), S_{v}=S \cap V\left(H^{v}+v\right)$ is a disjunctive dominating set of $H^{v}+v$. Let $u \in V\left(H^{v}+v\right) \backslash S_{v}$. Then $u \in V\left(H^{v}\right) \backslash S$. Since $S$ is a restrained disjunctive dominating set, there exists $w \in V(G \circ H) \backslash S$ such that $u w \in E(G \circ H)$ or there exist distinct $w, z \in V(G \circ H) \backslash S$ for which $d_{G \circ H}(u, w)=2=$ $d_{G \circ H}(u, z)$. In case the former holds, $w \in V\left(H^{v}\right) \backslash S_{v}$ and $u w \in E\left(H^{v}+v\right)$. If the latter holds, then $w$ and $z$ are distinct vertices in $V\left(H^{v}\right) \backslash S_{v}$ with $d_{H^{v}+v}(u, w)=2=d_{H^{v}+v}(u, z)$. Thus, $S_{v}$ is a restrained disjunctive dominating set of $H^{v}+v$. This proves $(i)(a)$. Now, suppose that $V\left(H^{v}\right) \backslash S \neq \varnothing$ and $\left|N_{G}(v) \backslash S\right|=1$. Let $u \in V\left(H^{v}\right) \backslash S$, and suppose that $\left|V\left(H^{v}\right) \backslash S\right|=1$. Since $S$ is a restrained disjunctive dominating set and $w \in S$ for all $w \in V(G \circ H)$ for which $u w \in E(G \circ H)$, there exist distinct $x, y \in V(G \circ H) \backslash S$ such that $d_{G \circ H}(u, x)=2=d_{G \circ H}(u, y)$. Necessarily, $x, y \in N_{G}(v)$, a contradiction. Thus $\left|V\left(H^{v}\right) \backslash S\right| \geq 2$, proving $(i)(b)$.

To prove (ii), let $v \in V(G) \backslash S$ for which $S \cap V\left(H^{v}\right) \neq \varnothing$. Since $S$ is a restrained disjunctive dominating set and $v \in V(G \circ H) \backslash S$, there exists $u \in V(G \circ H) \backslash S$ such that $u v \in E(G \circ H)$ or there exist distinct $x, y \in V(G \circ H) \backslash S$ for which $d_{G \circ H}(v, x)=2=$ $d_{G \circ H}(v, y)$. Note that for such $u$, either $u \in V\left(H^{v}\right)$ or $u \in N_{G}(v)$. That is, $(a)$ or $(b)$ holds. On the other hand, if $(a)$ and $(b)$ do not hold, $x, y \in V(G)$ and $d_{G}(x, v)=2=d_{G}(y, v)$ showing that $\left|N_{G}(v, 2) \cap[V(G) \backslash S]\right| \geq 2$.

Conversely, note first that because $S$ satisfies the properties in Theorem $5, S$ is a disjunctive dominating set of $G \circ H$. Let $u \in V(G \circ H) \backslash S$, and let $v \in V(G)$ such that $u \in V\left(H^{v}+v\right)$. Suppose that $u=v$. Then $u=v \in V(G) \backslash S$. If $S \cap V\left(H^{v}\right)=\varnothing$, then $u x \in E(G \circ H)$ for any $x \in V\left(H^{v}\right)$. Suppose that $S \cap V\left(H^{v}\right) \neq \varnothing$. Pick $x \in V\left(H^{v}\right) \backslash S$ or $x \in N_{G}(v) \backslash S$ in case $(i i)(a)$ or $(i i)(b)$ holds, respectively. Then $u x \in E(G \circ H)$. If $\left(\right.$ ii) (c) holds, then pick distinct $x, y \in V(G)$ for which $d_{G}(u, x)=2=d_{G}(u, y)$. Then $d_{G \circ H}(u, x)=2=d_{G \circ H}(u, y)$. Suppose that $u \in V\left(H^{v}\right)$. If $v \notin S$, then $v$ is the required vertex for which $u v \in E(G \circ H)$. Suppose that $v \in S$. Suppose further that $N_{G}(v) \subseteq S$. By $(i)(a)$, there exists $w \in V\left(H^{v}\right) \backslash S$ such that $u w \in E\left(H^{v}+v\right)$ or there exist distinct $w, z \in V\left(H^{v}\right) \backslash S$ for which $d_{H^{v}+v}(u, w)=2=d_{H^{v}+v}(u, z)$. This implies that there exists $w \in V(G \circ H) \backslash S$ such that $u w \in E(G \circ H)$ or there exist distinct $w, z \in V(G \circ H) \backslash S$ for which $d_{G \circ H}(u, w)=2=d_{G \circ H}(u, z)$. Finally, suppose that $N_{G}(v) \backslash S \neq \varnothing$. If $\left|N_{G}(v) \backslash S\right| \geq$ 
2, then there exist distinct $x, y \in N_{G}(v) \backslash S$ such that $d_{G \circ H}(u, x)=2=d_{G \circ H}(u, y)$. Suppose that $\left|N_{G}(v) \backslash S\right|=1$, say $x \in N_{G}(v) \backslash S$. We may also pick $y \in V\left(H^{v}\right) \backslash S$ with $u \neq y$ by $(i)(b)$. Then $u y \in E(G \circ H)$ or $d_{G \circ H}(u, y)=2=d_{G \circ H}(u, x)$. Accordingly, $S$ is a restrained disjunctive dominating set of $G \circ H$.

Corollary 6. For nontrivial connected graphs $G$ and $H, \gamma_{r}^{d}(G \circ H)=\gamma_{\times 2}(G)$.

Proof. Let $S \subseteq V(G)$ be a 2-dominating set of $G$. By Corollary 3, $S$ is a disjunctive dominating set of $G \circ H$. Moreover, since all the properties in Theorem 6 hold for $S, S$ is a restrained disjunctive dominating set of $G \circ H$. Thus, $\gamma_{r}^{d}(G \circ H) \leq|S|$. Since $S$ is arbitrary, $\gamma_{r}^{d}(G \circ H) \leq \gamma_{\times 2}(G)$. Further, using Corollary 3 again,

$$
\gamma_{\times 2}(G) \leq \gamma_{r}^{d}(G \circ H) \leq \gamma_{\times 2}(G)
$$

This proves the equality.

\subsection{On lexicographic product of graphs}

[15] For any graphs $G$ and $H$ and for any $C \subseteq V(G[H])$, there exists $S \subseteq V(G)$ for which $C=\cup_{x \in S}\left(\{x\} \times T_{x}\right)$, where $T_{x} \subseteq V(H)$ for each $x \in S$.

Theorem 7. [15] Let $G$ and $H$ be nontrivial connected graphs, and let $C=\cup_{x \in S}\left(\{x\} \times T_{x}\right)$. Then $C$ is a disjunctive dominating set of $G[H]$ if and only if one of the following holds:

(i) $S$ is a disjunctive total dominating set in $G$; or

(ii) $S$ is a distance-two dominating set of $G$ satisfying the following:

(a) For each $x \in V(G) \backslash N_{G}^{d}[S]$, where $N_{G}^{d}[S]=S \cup N_{G}^{d}(S)$, there exists $u \in S$ for which $d_{G}(u, x)=2$ and $\left|T_{u}\right| \geq 2$.

(b) For each $x \in S \backslash N_{G}(S, 2)$, either $T_{x}=\{y\}$ and is a dominating set of $H$ or $\left|T_{x}\right| \geq 2$.

Theorem 8. Let $G$ and $H$ be nontrivial connected graphs, and let $C=\cup_{x \in S}\left(\{x\} \times T_{x}\right)$ be a disjunctive dominating set of $G[H]$. Then $C$ is a restrained disjunctive dominating set of $G[H]$ if and only if $(i)$ or (ii) of Theorem 7 holds and each of the following holds for $S$ :

For each $x \in S \backslash\left(N_{G}(V(G) \backslash(S, 2))\right.$ for which $T_{x} \neq V(H)$,

(a) If $T_{u}=V(H)$ for all $u \in N_{G}(x, 2)$, then either $\left|V(H) \backslash T_{x}\right| \geq 3$ or $\left\langle V(H) \backslash T_{x}\right\rangle=K_{2}$.

(b) If $\left\langle V(H) \backslash T_{x}\right\rangle=\overline{K_{2}}$, then there exists $u \in N_{G}(x, 2)$ for which $T_{u} \neq V(H)$.

(c) If $\left|V(H) \backslash T_{x}\right|=1$, then one of the following holds: there exists $u \in N_{G}(x)$ for which $T_{u} \neq V(H)$; there exists $u \in N_{G}(x, 2)$ for which $\left|V(H) \backslash T_{u}\right| \geq 2$; there exist distinct $u, z \in N_{G}(x, 2)$ for which $T_{u} \neq V(H)$ and $T_{z} \neq V(H)$. 
Proof. Suppose that $C$ is a restrained disjunctive dominating set of $G[H]$. Since $C$ is a disjunctive dominating set of $G[H],(i)$ or $(i i)$ of Theorem 7 holds. Now, let $x \in S \backslash$ $\left(N_{G}(V(G) \backslash(S, 2))\right)$ for which $T_{x} \neq V(H)$. Then $u \in S$ for all $u \in N_{G}(x, 2)$. First, suppose that $T_{u}=V(H)$ for all $u \in N_{G}(x, 2)$. Pick $y \in V(H) \backslash T_{x}$. Then $(x, y) \notin C$ so that there exists $(u, v) \in V(G[H]) \backslash C$ such that $(x, y)(u, v) \in E(G[H])$ or there exist distinct vertices $(u, v),(z, w) \in V(G[H]) \backslash C$ for which $d_{G[H]}((x, y),(u, v))=2=d_{G[H]}((x, y),(z, w))$. Since $x \notin N_{G}(V(G) \backslash(S, 2))$, the preceding statement implies that $x=u$, in which case $v \in V(H) \backslash T_{x}$ and $y w \in E(H)$, or $u=x=z$ and $y, v$ and $w$ are distinct vertices in $V(H) \backslash T_{x}$. This establishes $(a)$.

Next, suppose that $\left\langle V(H) \backslash T_{x}\right\rangle=\overline{K_{2}}$. let $y, w \in\left\langle V(H) \backslash T_{x}\right\rangle$. Suppose that $(u, v) \in V(G[H]) \backslash C$ such that $(x, y)(u, v) \in E(G[H])$. Since $y, w \in\left\langle V(H) \backslash T_{x}\right\rangle$, it is necessary that $u \neq x$. Hence $u \in N_{G}(x)$ and $v \in V(H) \backslash T_{u}$. Consequently, $T_{u} \neq V(H)$. Suppose that $(u, v),(z, w) \in V(G[H]) \backslash C$ are distinct for which $d_{G[H]}((x, y),(u, v))=$ $2=d_{G[H]}((x, y),(z, w))$. The assumption implies that $u \in N_{G}(x, 2)$ or $z \in N_{G}(x, 2)$. If $u \in N_{G}(x, 2)$, then $T_{u} \neq V(H)$, and if $z \in N_{G}(x, 2)$, then $T_{z} \neq V(H)$. Hence, (b) holds.

Lastly, suppose that $\left|V(H) \backslash T_{x}\right|=1$, say $V\left(H \backslash T_{x}=\{y\}\right.$. If $(u, v) \in V(G[H]) \backslash C$ such that $(u, v)(x, y) \in E(G[H])$, then since $T_{x}=\{y\}, u \in N_{G}(x)$ and $T_{u} \neq V(H)$. On the other hand, if $(u, v),(z, w) \in V(G[H]) \backslash C$ are distinct for which $d_{G[H]}((x, y),(u, v))=$ $2=d_{G[H]}((x, y),(z, w))$, then $d_{G}(x, u)=2=d_{G}(x, z)$. If $u=z$, then $\left|V(H) \backslash T_{u}\right| \geq 2$. Otherwise, $T_{u} \neq V(H)$ and $T_{z} \neq V(H)$.

Conversely, suppose that Conditions $(i)$ or $(i i)$ of Theorem 7 holds. Then $C$ is a disjunctive dominating set of $G[H]$. Suppose further that all properties in Theorem 8 hold for $S$. Let $(x, y) \in V(G[H]) \backslash C$.

Case 1: If $x \notin S$, then pick $z \in V(H) \backslash\{y\}$ for which $z y \in E(H)$. In this case, $(x, z) \in$ $V(G[H]) \backslash C$ and $(x, y)(x, z) \in E(G[H])$.

Case 2: Suppose that $x \in S$. Then $y \notin T_{x}$. First, consider having $x \in N_{G}^{d}(V(G) \backslash$ $S)$. Suppose $u \in V(G) \backslash S$ such that $u x \in E(G)$. Pick $v \in V(H)$. Then $(u, v) \notin$ $C$ and $(x, y)(u, v) \in E(G[H])$. Suppose, on the other hand, that $u, z \in V(G) \backslash S$ for which $d_{G}(x, u)=2=d_{G}(x, z)$. Pick $v, w \in V(H)$. Then $(u, v),(z, w) \notin C$ and $d_{G[H]}((x, y),(u, v))=2=d_{G[H]}((x, y),(z, w))$. Next, suppose that $x \notin N_{G}^{d}(V(G) \backslash S)$. Suppose further that $x \in N_{G}(V(G) \backslash(S, 2))$. Pick $u \in V(G) \backslash S$ such that $1 \leq d_{G}(x, u) \leq$ 2. If $u x \in E(G)$, then $(u, v) \notin C$ and $(u, v)(x, y) \in E(G[H])$ for any $v \in V(H)$. Otherwise, pick distinct $v, w \in V(H)$. Then $(u, v)$ and $(u, w)$ are distinct vertices in $V(G[H]) \backslash C$ with $d_{G[H]}((x, y),(u, v))=2=d_{G[H]}((x, y),(u, w))$. Finally, suppose that $x \notin N_{G}(V(G) \backslash(S, 2))$. We consider the following subcases:

Subcase 2.1: Suppose that $T_{u}=V(H)$ for all $u \in N_{G}(x, 2)$. By Condition (a), either $\left|V(H) \backslash T_{x}\right| \geq 3$ or $\left\langle V(H) \backslash T_{x}\right\rangle=K_{2}$. Let $y, w \in V(H) \backslash T_{x}$. If $\left|V(H) \backslash T_{x}\right| \geq 3$ and $v, w \in\left(V(H) \backslash T_{x}\right) \backslash\{y\}$ are distinct, then $(x, v)$ and $(x, w)$ are distinct vertices in $V(G[H]) \backslash C$ with $d_{G[H]}((x, y),(x, v)) \leq 2$ and $d_{G[H]}((x, y),(x, w)) \leq 2$. On the other hand, if $y, w \in\left\langle V(H) \backslash T_{x}\right\rangle=K_{2}$, then $(x, w) \in V(G[H]) \backslash C$ with $(x, y)(x, w) \in E(G[H])$.

Subcase 2.2: Suppose that $T_{u} \neq V(H)$ for some $u \in N_{G}(x, 2)$. If $\left|V(H) \backslash T_{x}\right| \geq 3$, then, 
as done in Subcase 2.1, there exists $(x, v) \in V(G[H]) \backslash C$ such that $(x, y)(x, v) \in E(G[H])$ or there exist distinct $(x, v),(x, w) \in V(G[H]) \backslash C$ for which $d_{G[H]}((x, y),(x, v))=2=$ $d_{G[H]}((x, y),(x, w))$. Suppose that $y w \in\left\langle V(H) \backslash T_{x}\right\rangle=K_{2}$. Then $(x, w) \in V(G[H]) \backslash C$ with $(x, y)(x, w) \in E(G[H])$. If $y w \in\left\langle V(H) \backslash T_{x}\right\rangle=\overline{K_{2}}$, then there exists $u \in N_{G}(x, 2)$ for which $T_{u} \neq V(H)$, by Condition $(b)$. Here, $d_{G[H]}\left((x, y),(x, w)=2\right.$. Pick $v \in V(H) \backslash T_{u}$. Then $(u, v) \notin C$ and $d_{G[H]}\left((x, y),(u, v) \leq 2\right.$. Finally, suppose that $\left|V(H) \backslash T_{x}\right|=1$. By Condition (c), one of the following holds: there exists $u \in N_{G}(x)$ for which $T_{u} \neq V(H)$; there exists $u \in N_{G}(x, 2)$ for which $\left|V(H) \backslash T_{x}\right| \geq 2$; there exist distinct $u, z \in N_{G}(x, 2)$ for which $T_{u} \neq V(H)$ and $T_{z} \neq V(H)$. If $u \in N_{G}(x)$ and $v \in V(H) \backslash T_{u}$, then $(u, v) \notin C$ and $(x, y)(u, v) \in E(G[H])$. If $u \in N_{G}(x, 2)$ for which $\left|V(H) \backslash T_{u}\right| \geq 2$ and $v, w \in V(H) \backslash T_{u}$ are distinct, then $(u, v)$ and $(u, w)$ are distinct in $V(G[H]) \backslash C$ and $d_{G[H]}((x, y),(u, v)) \leq 2$ and $d_{G[H]}((x, y),(u, w)) \leq 2$. If $u, z \in N_{G}(x, 2)$ are distinct for which $T_{u} \neq V(H)$ and $T_{z} \neq V(H)$, then $(u, v)$ and $(z, w)$ are distinct vertices in $V(G[H]) \backslash C$ for all $v \in V(H) \backslash T_{u}$ and $w \in V(H) \backslash T_{z}$ with $d_{G[H]}((x, y),(u, v))=2=d_{G[H]}((x, y),(z, w))$.

Accordingly, $C$ is a restrained disjunctive dominating set of $G[H]$.

Corollary 7. Let $G$ and $H$ be nontrivial connected graphs. Then

$$
\gamma_{r}^{d}(G[H]) \leq \min \left\{\gamma_{t}^{d}(G), 2 \gamma_{2}(G)\right\}
$$

Proof. Let $S \subseteq V(G)$ be a disjunctive total dominating set of $G$, and let $u \in V(H)$. Then $S \times\{u\}=\cup_{x \in S}(\{x\} \times\{u\})$ is a disjunctive dominating set of $G[H]$ by Theorem 7. Since $S$ satisfies condition (ii) of Theorem $8, S$ is a restrained disjunctive dominating set of $G[H]$. Consequently, $\gamma_{r}^{d}(G[H]) \leq|S|$. Since $S$ is arbitrary, $\gamma_{r}^{d}(G[H]) \leq \gamma_{t}^{d}(G)$. Similar arguments as above, if $S \subseteq V(G)$ is a distance-two dominating set of $G$ satisfying condition (ii) of Theorem 7, then $2 S$ is a restrained disjunctive dominating set of $G[H]$. Thus, $\gamma_{r}^{d}(G[H]) \leq 2 \gamma_{2}(G)$. Therefore, $\gamma_{r}^{d}(G[H]) \leq \min \left\{\gamma_{t}^{d}(G), 2 \gamma_{2}(G)\right\}$.

Since $\gamma_{r}^{d}\left(C_{7}\left[P_{8}\right]\right)=\gamma_{t}^{d}\left(C_{7}\right)=2 \gamma_{2}\left(C_{7}\right)=4$, the inequality in Corollary 7 is sharp.

\section{Conclusion}

Let $G$ and $H$ be nontrivial connected graphs. It is shown that the restrained disjunctive domination number in the join of two graphs $(G+H)$ is the $\min \{\gamma(G), \gamma(H), 2\}$ and the restrained disjunctive domination number in the corona of two graphs $(G \circ H)$ is the $\left(\gamma_{\times 2}(G)\right)$. Under the same condition, the minimum of the sets between disjuntive total domination number of graph $G$ and twice of distance-2 domination number of graph $G$, denoted by $\min \left\{\gamma_{t}^{d}(G), 2 \gamma_{2}(G)\right\}$ proves to be a sharp bound for the restrained disjunctive domination number of the lexicographic product of graphs $G$ and $H$. 


\section{Acknowledgements}

This research was undertaken at the Technische Universitat Kaiserslautern, Germany, under the Faculty and Student Exchange Program of the project "Graph Theory and Optimization (GraThO) with Applications in Industry and Society" and funded by the Zamboanga Peninsula Polytechnic State University, Philippines. Also, The authors would like to recognize the efforts of the anonymous reviewers whose suggestions and recommendations contributed to the big improvement of the paper.

\section{References}

[1] C. Berge. Theorie des graphes et ses applications. Dunod, Paris, 1958.

[2] F. Buckley and F. Haray. Distance in Graphs. Addison-Wesley Publishing Company, Redwood City, 1990.

[3] M. Chellali. Bounds on the 2-domination number in cactus graphs. Opuscula Mathematica, 26:5-12, 2006.

[4] E. Cockayne and S. Hedetniemi. Towards a theory of domination in graphs. Networks, $7(3): 247-261,1977$.

[5] Dankelmann, D. Day, D. Erwin, S. Mukwembi, and H. Swart. Domination with exponential decay. Discrete Mathematics, 309:5877 - 5883, 2009.

[6] G.S. Domke, J.H. Hatting, S.T. Hedetniemi, R.C. Laskar, and L.R. Markus. Restrained domination in graphs. Discrete Mathematics, 203:61-69, 1999.

[7] B.D. Domolan and S.R. Canoy Jr. 2-domination and restrained 2-domination in graphs. Applied Mathematical Sciences, 9:5651-5659, 2015.

[8] W. Goddard, M.A. Henning, and C.A. McPillan. The disjunctive domination number of a graph. Quaestiones Mathematicae, 37:547-561, 2014.

[9] A. Hansberg and L. Volkmann. Note on graphs with equal domination and 2domination numbers. Discrete Mathematics, 308:2277-2281, 2008.

[10] T.W. Haynes, S.T. Hedetniemi, and P.J. Slater. Fundamentals of Domination in Graphs. Marcel Dekker, Inc., New York, 1998.

[11] S.M. Hedetniemi, S.T. Hedetniemi, J. Knisely, and D.F. Rall. Secondary domination in graphs. AKCE Int. J. Graphs Comb., 5:103-115, 2008.

[12] M.A. Henning and V. Naicker. Graphs with large disjunctive total domination number. Discrete Mathematics, 17:255-282, 2015.

[13] M.A. Henning and V. Naicker. Bounds on the disjunctive total domination number of a tree. Discussiones Mathematicae, 36:153 - 171, 2016. 
[14] M.A. Henning and V. Naicker. Disjunctive total domination in graphs. Comb. Opti., 31:1090-1110, 2016.

[15] F. Jamil and R. Malalay. On disjuntive domination in graphs. Quaestiones Mathematicae, 43:149 - 168, 2019.

[16] G. Kokilambal and K Kayathri. Restrained domination in trees. International Journal of Applied Engineering Research, 14:0973-4562, 2020.

[17] G.B. Monsanto and H.M. Rara. Resolving restrained domination in graphs. European Journal of Pure and Applied Mathematics, 14:829-841, 2021.

[18] O. Ore. Theory of graphs. Amer. Math. Soc. Colloq. Publ., Chicago, 1962.

[19] M.N. Paspasan and S.R. Canoy Jr. Restrained total edge domination in graphs. Applied Mathematical Sciences, 9:7139-7148, 2015.

[20] N. Sridharan, V.S.A. Subramanian, and M.D. Elias. Bounds on the distance twodomination number of a graph. Graphs and Combinatorics (Springer-Verlag), 18:667 -675, 2002. 